\title{
Tunable Low Threshold Optical Tristability at Terahertz Frequencies via a Pair of Parallel Graphene Layers' Configuration
}

\author{
Yunyang Ye, ${ }^{1}$ Jiao Tang, ${ }^{1}$ Zhiwei Zheng $\left(\mathbb{D},{ }^{1}\right.$ Hui Zhou, ${ }^{2}$ Minzhu Xie, \\ Leyong Jiang $\mathbb{D}^{1}{ }^{1}$ and Shengyou Qian $\mathbb{D}^{1}$ \\ ${ }^{1}$ College of Physics and Electronic Science, Hunan Normal University, Changsha 410081, China \\ ${ }^{2}$ College of Information Science and Engineering, Hunan Normal University, Changsha 410081, China \\ Correspondence should be addressed to Leyong Jiang; jiangly28@hunnu.edu.cn and Shengyou Qian; 155045943@qq.com
}

Received 4 April 2018; Accepted 28 August 2018; Published 18 September 2018

Academic Editor: Qinghua Guo

Copyright (c) 2018 Yunyang Ye et al. This is an open access article distributed under the Creative Commons Attribution License, which permits unrestricted use, distribution, and reproduction in any medium, provided the original work is properly cited.

We investigate theoretically the optical tristability of transmission at a pair of parallel graphene layers system. We discuss the influence of the graphene sheets on the hysteretic response of the TE-polarized transmitted light. It is demonstrated that the optical tristability in this configuration can be realized due to the giant third-order nonlinear conductivity of graphene and appropriate structure parameters. The tristable behavior of the transmitted light can be controlled via suitably varying the Fermi energy of the graphene. Besides, the optical tristable behavior is strongly dependent on the relaxation time of the graphene and the dispersion characteristics of the surrounding dielectrics. Moreover, the threshold of the optical tristability can be reduced by appropriately increasing the number of graphene layers, making this simple structure a good candidate for dynamic tunable and low threshold optical tristable device in the terahertz $(\mathrm{THz})$ frequencies.

\section{Introduction}

Optical bistability and multistability devices are widely used in the areas of optoelectronics such as optical memory, optical transistor, all-optical logic gate, and all-optical switching due to their ability of harnessing optical nonlinear characteristics [1-4]. The generation and control means of optical multistability, especially related optical multistable device play a key role in optical communication system and optical network. In particular, the key to solving transmission and processing of information is micro/nano-optical multistable devices integrated to chip size. Therefore, people proposed various ways and methods to realize the generation and control of optical bistability such as Fabry-Perot cavities [5], nonlinear prism coupler [6], subwavelength metallic gratings [7], and semiconductor quantum well systems [8]. The phenomenon of optical multistability of four-level cascade-type cold atoms [9], semiconductor quantum well nanostructure [10], and polaritonic materials doped with nanoparticles [11] have also raised heated research. However, producing obvious optical nonlinearities effect requires high incident power owing to low nonlinear refractive index of traditional nonlinear materials; thus optical bistable device with longer size and larger incident light intensity is required to generate optical nonlinearities effect. Thus the lack of competitive advantage in high-density integrated optical circuits and its applications are limited by these requirements. Optical multistability in new materials and novel structure has become the main research direction in the field of optical multistability.

Recently, graphene, a two-dimensional atomic crystal material, has attached much attention in the areas of optical, material science, and physics owing to its various outstanding characteristics [12-14]. The partial characteristic of graphene can give full play to its advantage in the field of optical multistability. For instance, strong nonlinear optical absorption coefficient of graphene [15], fast modulation speed [16], broadband [17], the strong interaction between light and graphene [18], and the optical property are easy to control dynamically [19]. Graphene has great potential in the field of optical multistability due to these advantages. Graphene-based 


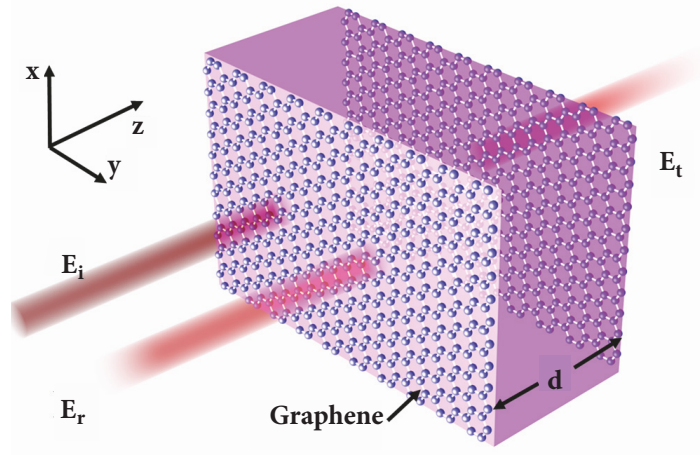

FIGURE 1: Schematic diagram of a pair of parallel graphene layers with the insertion of dielectric slab. A plane wave of amplitude $E_{i}$ is incident on the structure, giving rise to a reflected and a transmitted wave with amplitudes $E_{r}$ and $E_{t}$, respectively.

optical bistability and multistability begin to attract much attention as well. For example, Gu et al. have realized ultralow power resonant optical bistability in graphene-silicon hybrid optoelectronic devices for the first time [20]. Horvath et al. have observed the phenomenon of optical bistability and enhanced thermal nonlinearity in a graphene-silicon waveguide resonator [21]. Bao et al. researched optical bistability in a Fabry-Perot cavity containing monolayer and bilayer graphene which have been observed optical bistability and experimentally realized graphene-based fast optical bistability [22]. More recently, Yin et al. proposed a single dielectric layer sandwiched between two nonlinear graphene layers to realize the controllable transition between optical bistability and multistability [23].

In this paper, we have theoretically investigated the optical tristability with low threshold by constructing a simple double layer graphene structure. The optical tristability mainly originates from the huge third-order nonlinear coefficient of graphene and the suitable structural parameters. The conductivity of graphene can be tuned dynamically by external electric field. Graphene-based optical devices with intrinsic optical tristability allow us to explore the promise of using such elements as the building block of future integrated optics and digital all-optical circuitry.

\section{Theoretical Model and Method}

We consider a structure composed of dielectric slab (with thickness $d$ and refractive index $n$ ) and graphene; both sides of dielectric slab are covered by monolayer graphene, as shown in Figure 1, where $z$ direction is perpendicular to the plane of the monolayer graphene and a plane wave of amplitude $E_{i}$ is normally incident on the structure, giving rise to a reflected and a transmitted wave with amplitudes $E_{r}$ and $E_{t}$. We assumed incident wavelength $\lambda=300 \mu \mathrm{m}$ and dielectric slab is silicon with refractive index $n=11.9$ in $\mathrm{THz}$ frequency range; the thickness of dielectric slab satisfies $d=\lambda /(4 n)$. A plane wave of amplitude $E_{i}$ is normally incident on the structure. The linear conductivity of graphene in $\mathrm{THz}$ range can be expressed as [24]

$$
\sigma_{0}=\frac{i e^{2} E_{F}}{\pi \hbar^{2}(\omega+i / \tau)}
$$

where $E_{F}$ is the Fermi energy of graphene, $\tau$ is the relaxation time, and $\omega$ is the frequency. $e$ and $\hbar$ are the universal constants related to the electron charge and reduced Planck's constant, respectively. From formula (1), we can see that the conductivity of graphene can be used to describe its optical properties. It is noteworthy that graphene third-order nonlinear conductivity is not negligible when the intensity is enough to a certain extent, and the third-order conductivity of graphene in $\mathrm{THz}$ band can be described as $[25,26]$

$$
\sigma_{3}=-i \frac{9}{8} \frac{e^{4} \nu_{F}^{2}}{\pi \hbar^{2} E_{F} \omega^{3}}
$$

where $v_{F}=10^{6} \mathrm{~m} / \mathrm{s}$ is the Fermi velocity of electrons. In the presence of the nonlinearity, the optical conductivity of graphene $\sigma=\sigma_{0}+\sigma_{3}|E|^{2}$ and $\mathrm{E}$ is the tangential electric field of the electromagnetic wave at the interface.

Next, we use the modified transmission matrix to calculate the transmittance of the structure to get the relation of $E_{t}$ and $E_{i}$ [27]. In this paper, we suppose that incident electromagnetic wave is TE-polarized. Therefore, the transmission matrix on adjacent dielectric surfaces can be expressed as

$$
D_{12}=\frac{1}{2}\left[\begin{array}{cc}
1+\eta_{T E}+\xi_{T E} & 1-\eta_{T E}+\xi_{T E} \\
1-\eta_{T E}-\xi_{T E} & 1+\eta_{T E}-\xi_{T E}
\end{array}\right]
$$

in this equation, $\eta_{T E}=k_{2 z} / k_{1 z}, \xi_{T}=\sigma \mu_{0} \omega / k_{1 z}$, where $k_{1 z}$ and $k_{2 z}$ represent the components of wave vectors $k_{1}$ and $k_{2}$ in the transmission direction of electromagnetic wave, respectively. Combining with the propagation matrix of dielectric slab, we can get the transmission coefficient of the structure. Besides, the relation between $E_{i}$ and $E_{t}$ can be described as

$$
\left|\frac{E_{\mathrm{t}}}{E_{\mathrm{i}}}\right|=\left|\frac{1}{\left(1+\eta_{\mathrm{ad}}+\xi_{\mathrm{ad}}\right)\left(1+\eta_{\mathrm{da}}+\xi_{\mathrm{da}}\right) e^{-i k_{d z} d}+\left(1-\eta_{\mathrm{ad}}+\xi_{\mathrm{ad}}\right)\left(1-\eta_{\mathrm{da}}-\xi_{\mathrm{da}}\right) e^{i k_{d z} d}}\right|,
$$

where $k_{d z}$ represents the component of wave vectors $k_{d}$ in the transmission direction of electromagnetic wave in dielectric slab.

\section{Results and Discussions}

In this section, we will discuss the phenomenon of optical tristability caused by the nonlinear graphene in the structure. 


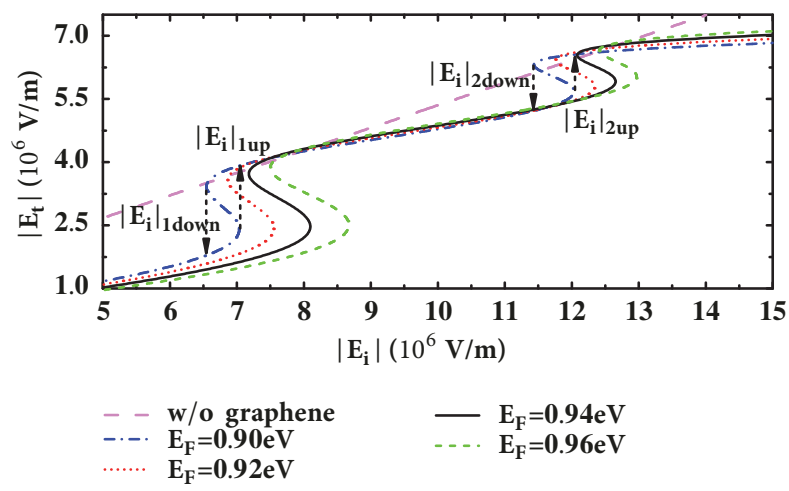

(a)

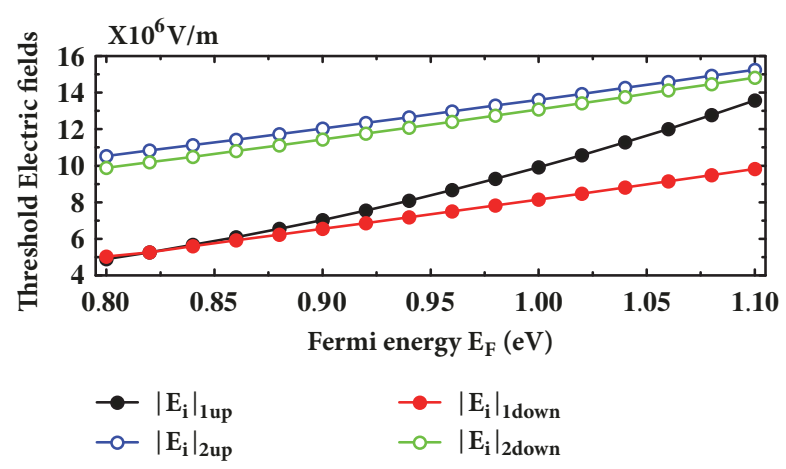

(b)

FIGURE 2: (a) Schematic diagram of the transmitted electric field as a function of the incident electric field for different Fermi energies $E_{F}$ of the graphene. (b) Schematic diagram of the switch-up and switchup threshold electric field as a function of $E_{F}$.

Based on the method of transfer matrix above, the characteristics of transmission of the structure can be obtained; thus the relation of incident electric field $\mathrm{E}_{\mathrm{i}}$ to transmitted electric field $\mathrm{E}_{t}$ can be conveniently presented, as shown in Figure 2(a). We set the relaxation time of graphene as $\tau=$ $1.25 \mathrm{ps}$; the phenomenon of optical bistability and optical multistability are strongly dependent on the third-order or multiorder nonlinear coefficients; thus the huge thirdorder nonlinear conductivity of graphene is a very important factor for realizing optical tristability in the structure. When the graphene does not exist, the tristable curve cannot be achieved under some conditions. However, the addition of graphene makes a big difference and the hysteresis of optical tristability can be clearly produced. Due to the huge third-order nonlinear conductivity of graphene, multivalued phenomenon between incident electric field and transmitted electric field can be satisfied. Here we take $E_{F}=0.90 \mathrm{eV}$ as an example; the transmitted electric field $E_{t}$ gradually increase with the increase of $E_{i}$ when the incident electric field $E_{i}$ is at a small value; this is the first stable state. However, the transmitted electric field switches to the second stable state when $E_{i}$ is continuously enhanced to $\left|E_{i}\right|_{1 \text { up }}=7.03 \times$ $10^{6} \mathrm{~V} / \mathrm{m}$; at the moment, reducing the incident electric field $E_{i}$, the transmitted electric field will not immediately return to the first stable state but will decrease slowly in

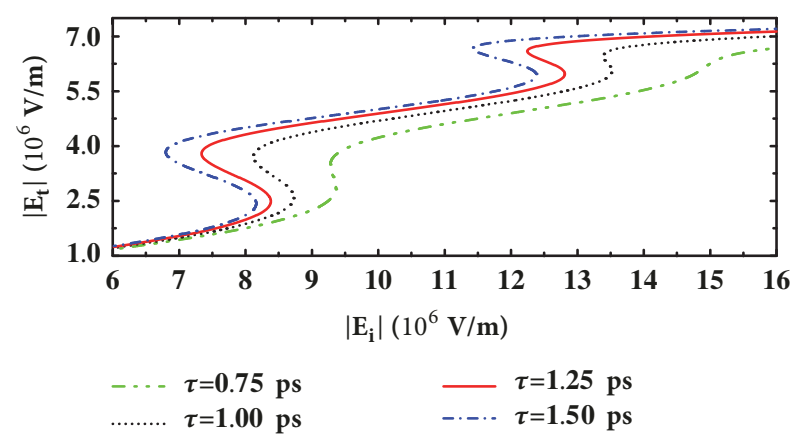

(a)

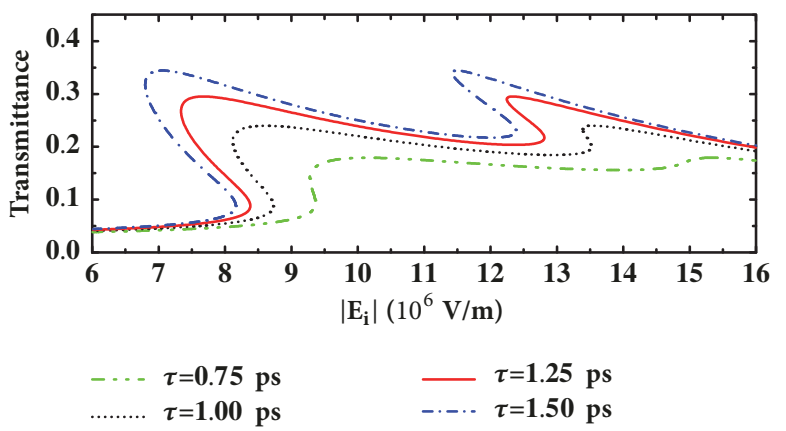

(b)

FIGURE 3: A diagram of the relationship between transmitted electric field (a) and transmittance (b) with incident electric field under different relaxation times $\tau$. The other parameters are the same as before.

the second stable state; the second stable state will jump to the first stable state until the incident electric field drops to $\left|E_{i}\right|_{1 \text { down }}=6.55 \times 10^{6} \mathrm{~V} / \mathrm{m}$, and hysteresis width satisfies $\Delta\left|E_{i}\right|=\left|E_{i}\right|_{1 \text { up }}-\left|E_{i}\right|_{1 \text { down }}=0.48 \times 10^{6} \mathrm{~V} / \mathrm{m}$. If the incident electric field $E_{i}$ continuously increase in the second stable state, the transmitted electric field $E_{i}$ will continue to increase slowly and synchronously; the transmitted stable state will jump from the second stable state to the third stable state until $E_{i}$ continues to be enhanced to $\left|E_{i}\right|_{2 \text { up }}=$ $12.03 \times 10^{6} \mathrm{~V} / \mathrm{m}$. After entering the third stable state, if we reduce $E_{i}, E_{t}$ will jump from the third stable state to the second stable state when $\left|E_{i}\right|_{2 \text { down }}=11.44 \times 10^{6} \mathrm{~V} / \mathrm{m}$, and the hysteresis width satisfies $\Delta\left|E_{i}\right|=\left|E_{i}\right|_{2 \text { up }}-\left|E_{i}\right|_{2 \text { down }}=$ $0.59 \times 10^{6} \mathrm{~V} / \mathrm{m}$. This is a very typical phenomenon of optical tristability and the nonlinear property of graphene plays a key role in the typical phenomenon. More importantly, the electrically tunable conductivity of graphene also provides flexible and tunable properties for optical tristability. At the moment, we plotted the hysteresis of the structure at different Fermi energies, as shown in Figure 3(a). We can see that the threshold of two stable states was significantly reduced with the gradually decrease of the graphene Fermi energies from $0.96 \mathrm{eV}$ to $0.92 \mathrm{eV}$. In order to more clearly illustrate the phenomenon, the effect of Fermi energy changes on the threshold and hysteresis width is listed in detail; with the decrease of Fermi energy of graphene, we can clearly see that the threshold of tristability obviously decreases and the 
hysteresis width between the first stable state and the second stable state will gradually decrease; when Fermi energy is reduced to $0.825 \mathrm{eV}$, the hysteresis width will be zero and the tristability will switch to bistability, as shown in Figure 3(b). However, the hysteresis width between the second stable state and the third stable state is hardly changing with the change of Fermi energy. Therefore, Fermi energy can not only manipulate the threshold of tristability but also affect the hysteresis width. It also provides a feasible idea for realizing flexible and tunable optical bistable and tristable device.

According to formulas (1) and (2), it is found that the relaxation time $\tau$ of graphene is also an important parameter affecting the conductivity of graphene, although it only affects the linear part of the conductivity of the graphene, that is, the real part of conductivity of graphene. It also provides a method for manipulating the characteristic of optical tristability. We presented the influence of changes of the relaxation time $\tau$ of graphene on optical tristability; as shown in Figure 3, it can be seen that the relaxation time $\tau$ of graphene plays a key role in the multivalued phenomenon of transmitted electric field. When the relaxation time $\tau=1.25 \mathrm{ps}$, because the discriminant of the incident electric field $E_{i}$ deviating from the transmitted electric field $E_{t}$ satisfies $\Delta>0$, there is an obvious multivalue phenomenon near the incident electric field $\left|E_{i}\right|=8 \times$ $10^{6} \mathrm{~V} / \mathrm{m}$ and $\left|E_{i}\right|=13 \times 10^{6} \mathrm{~V} / \mathrm{m} ;$ a similar situation also appears in the curve of the transmittance changes with the incident electric field, as shown in Figure 3(b). However, when the relaxation time gradually decreases, $\Delta>$ 0 in the discriminant where the incident electric field $E_{i}$ deviating from the transmitted electric field $E_{t}$ is gradually not satisfied, resulting in a gradual decrease in the hysteresis width, and eventually the stable state disappears. Thus it can be seen that the relaxation time of graphene has a great advantage in manipulating the hysteresis width and threshold of optical tristability. However, it is noteworthy that the relaxation time $\tau$ of graphene is not easily controlled. Once the sample is prepared, its value is not easy to regulate any more.

In order to further research and understand the characteristics of multistability, we also discussed the influence of dispersion characteristic of the dielectric slab on the optical tristability, as shown in Figure 4. We showed the effect of changes of dielectric constant and the thickness on the optical tristability, respectively. We can see from Figure 4(a) that there is an obvious difference on the effect of dielectric constant on the two hysteresis widths. With the increase of dielectric constant, the second hysteresis width $\Delta\left|E_{i}\right|=$ $\left|E_{i}\right|_{2 \text { up }}-\left|E_{i}\right|_{2 \text { down }}$ can be enhanced, but the first hysteresis width $\Delta\left|E_{i}\right|=\left|E_{i}\right|_{\text {up }}-\left|E_{i}\right|_{1 \text { down }}$ can be obviously suppressed. This parameter plays a key role in designing optical multistable devices when the hysteresis width is needed to be controlled. Apart from dielectric constant, the thickness of dielectric slab has similar effect on the optical tristability; it is found that the increase of thickness of dielectric slab can inhibit the second hysteresis width: $\Delta\left|E_{i}\right|=\left|E_{i}\right|_{2 \text { up }}-\left|E_{i}\right|_{\text {2down }}$; at the same time, the first hysteresis width $\Delta\left|E_{i}\right|=\left|E_{i}\right|_{\text {up }}-$ $\left|E_{i}\right|_{1 \text { down }}$ will be obviously increased.

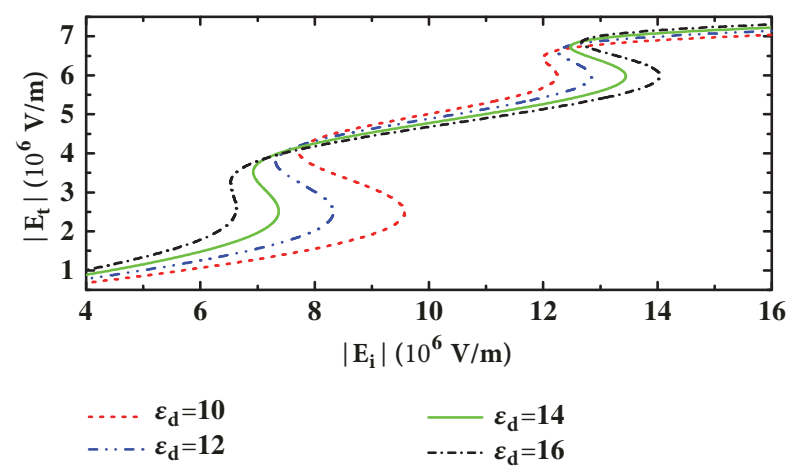

(a)

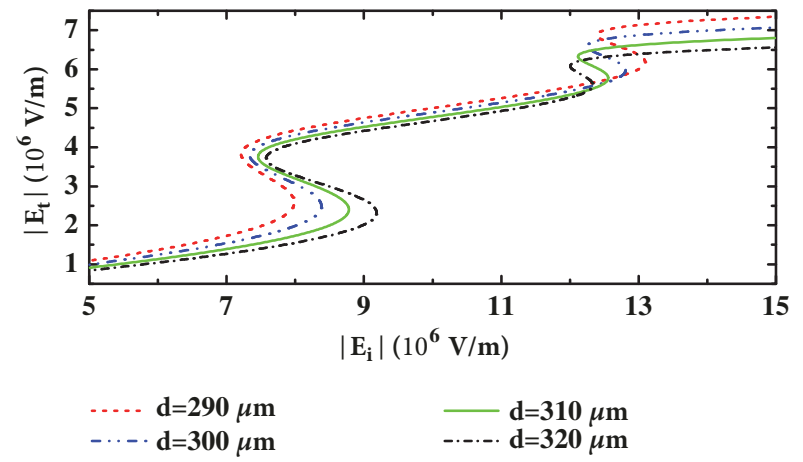

(b)

FIGURE 4: Schematic diagram of the influence of the dielectric constant (a) and the thickness (b) of the dielectric plate on the tristable phenomenon. The other parameters are the same as before.

The parameters of the structure provide more flexible choices for hysteresis characteristic of optical multistability.

It is known that that the phenomenon of multistability of the structure is strongly related to the third-order nonlinear conductivity of graphene. The threshold value of optical multistability not only closely relates to the third-order nonlinear conductivity of graphene but also directly influences the practical application of optical multistability devices. Therefore, it is necessary to reduce the threshold value as low as possible. Based on the above, we consider a pathway to further reduce the threshold of optical multistability on the structure of Figure 1. In order to realize the further decrease of threshold of optical tristability, we embed the monolayer graphene and combine appreciate parameters; the results are shown in Figure 5. At the new structure, we set the Fermi energy of graphene $E_{F}=0.8 \mathrm{eV}$ and the relaxation time of graphene $\tau=1.5 \mathrm{ps}$; the constants of dielectric slab 1 and dielectric slab 2 are set as 11.9 and 3.84, respectively. The situation of double-layered graphene has been shown in the figure as well. In contrast, it is found that the typical phenomenon of optical tristability can also occur under the novel structure. However, compared with the double-layered graphene, the threshold of hysteresis response is significantly reduced. The phenomenon not only indicates the advantage of reducing the threshold of optical bistability and optical tristability owing to the increase of graphene layers but also provides a feasible way for realizing optical multistability. 


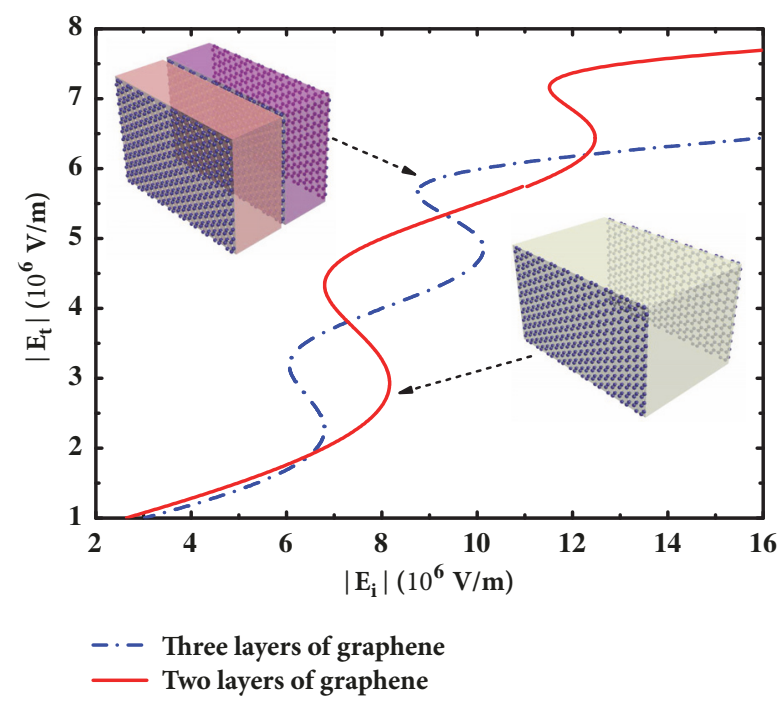

FIGURE 5: Optical tristability diagram of the system with two layers of graphene (red solid lines) and three layers of graphene (bluedotted).

\section{Conclusions}

In conclusion, we theoretically investigated the flexible and controllable optical tristability in the simple structure of monolayer graphene covering both sides of the dielectric slab. It is found that the optical tristability can occur in the structure by selecting appropriate parameters. The key to generating optical tristability is the huge third nonlinear conductivity of graphene. We can flexibly manipulate hysteretic response characteristic optical tristability by tuning the dispersion parameters of graphene. Furthermore, we have simply verified embedding monolayer graphene in the structure; not only can we create conditions for achieving optical multistability but also we can further reduce the optical tristable threshold by combining suitable structural parameters. We believe that simple and controllable optical tristability and optical multistability have a potential application in optical switch, optical limiting, and other optical devices.

\section{Data Availability}

No additional data are available.

\section{Conflicts of Interest}

The authors declare that they have no conflicts of interest.

\section{Acknowledgments}

This work is partially supported by the National Natural Science Foundation of China (Grant nos. 11474090, 11704119, 61772197, 61701180, and 11647135), the Hunan Provincial Natural Science Foundation of China (Grant nos. 2018JJ3325, 2016JJ6097, and 14JJ6007), the Scientific Research Fund of Hunan Provincial Education Department (Grant nos.
17C0945 and 17C0957), and the Project Supported for excellent talents in Hunan Normal University (Grant no. ET1502).

\section{References}

[1] H. Nihei and A. Okamoto, "Switching time of optical memory devices composed of photonic crystals with an impurity threelevel atom," Japanese Journal of Applied Physics, vol. 40, no. 12, pp. 6835-6840, 2001.

[2] G. Assanto, Z. Wang, D. J. Hagan, and E. W. Vanstryland, “Alloptical modulation via nonlinear cascading in type II secondharmonic generation," Applied Physics Letters, vol. 67, p. 2120, 1995.

[3] H. Tsuda and T. Kurokawa, "Construction of an all-optical flipflop by combination of two optical triodes," Applied Physics Letters, vol. 57, no. 17, pp. 1724-1726, 1990.

[4] D. A. Mazurenko, R. Kerst, J. I. Dijkhuis et al., "Ultrafast Optical Switching in Three-Dimensional Photonic Crystals," Physical Review Letters, vol. 91, no. 21, 2003.

[5] A. Grieco, B. Slutsky, D. T. H. Tan, S. Zamek, M. P. Nezhad, and Y. Fainman, "Optical bistability in a silicon waveguide distributed bragg reflector Fabry-pérot resonator," Journal of Lightwave Technology, vol. 30, no. 14, pp. 2352-2355, 2012.

[6] G. I. Stegeman, G. Assanto, R. Zanoni et al., "Bistability and switching in nonlinear prism coupling," Applied Physics Letters, vol. 52, no. 11, pp. 869-871, 1988.

[7] C. Min, P. Wang, C. Chen et al., "All-optical switching in subwavelength metallic grating structure containing nonlinear optical materials," Optics Expresss, vol. 33, no. 8, pp. 869-871, 2008.

[8] J. Li, X. Hao, J. Liu, and X. Yang, "Optical bistability in a triple semiconductor quantum well structure with tunnellinginduced interference," Physics Letters A, vol. 372, no. 5, pp. 716$720,2008$.

[9] H. R. Hamedi, A. Khaledi-Nasab, A. Raheli, and M. Sahrai, "Coherent control of optical bistability and multistability via double dark resonances (DDRs)," Optics Communications, vol. 312, pp. 117-122, 2014.

[10] S. Hossein Asadpour and H. Rahimpour Soleimani, "Optical bistability and multistability via biexciton coherence in semiconductor quantum well nanostructure," Optics Communications, vol. 315, pp. 347-351, 2014.

[11] Z. Wang and B. Yu, "Optical bistability and multistability in polaritonic materials doped with nanoparticles," Laser Physics Letters, vol. 11, no. 11, p. 115903, 2014.

[12] A. K. Geim and K. S. Novoselov, “The rise of graphene," Nature Materials, vol. 6, no. 3, pp. 183-191, 2007.

[13] A. H. Castro Neto, F. Guinea, N. M. R. Peres, K. S. Novoselov, and A. K. Geim, "The electronic properties of graphene," Reviews of Modern Physics, vol. 81, no. 1, pp. 109-162, 2009.

[14] K. S. Novoselov, V. I. Fal'Ko, L. Colombo, P. R. Gellert, M. G. Schwab, and K. Kim, "A roadmap for graphene," Nature, vol. 490, no. 7419, pp. 192-200, 2012.

[15] E. Hendry, P. J. Hale, J. Moger, A. K. Savchenko, and S. A. Mikhailov, "Coherent nonlinear optical response of graphene," Physical Review Letters, vol. 105, no. 9, Article ID 097401, 2010.

[16] J.-H. Chen, C. Jang, S. Xiao, M. Ishigami, and M. S. Fuhrer, "Intrinsic and extrinsic performance limits of graphene devices on $\mathrm{SiO}_{2}$," Nature Nanotechnology, vol. 3, no. 4, pp. 206-209, 2008. 
[17] F. Bonaccorso, Z. Sun, T. Hasan, and A. C. Ferrari, "Graphene photonics and optoelectronics," Nature Photonics, vol. 4, no. 9, pp. 611-622, 2010.

[18] F. H. L. Koppens, D. E. Chang, and F. J. García de Abajo, "Graphene plasmonics: a platform for strong light-matter interactions," Nano Letters, vol. 11, no. 8, pp. 3370-3377, 2011.

[19] Z. Q. Li, E. A. Henriksen, Z. Jiang et al., "Dirac charge dynamics in graphene by infrared spectroscopy," Nature Physics, vol. 4, no. 7, pp. 532-535, 2008.

[20] T. Gu, N. Petrone, J. F. McMillan et al., "Regenerative oscillation and four-wave mixing in graphene optoelectronics," Nature Photonics, vol. 6, no. 8, pp. 554-559, 2012.

[21] C. Horvath, D. Bachman, R. Indoe, and V. Van, "Photothermal nonlinearity and optical bistability in a graphene-silicon waveguide resonator," Optics Expresss, vol. 38, no. 23, pp. 5036-5039, 2013.

[22] Q. Bao, J. Chen, Y. Xiang et al., "Graphene nanobubbles: a new optical nonlinear material," Advanced Optical Materials, vol. 3, no. 6, pp. 744-749, 2015.

[23] L. Guo, Y. He, Y. Chen, and C. Yin, "Controllable transition between optical bistability and multistability in graphene/dielectric/graphene structure," The European Physical Journal B, vol. 91, no. 5, Paper No. 79, 7 pages, 2018.

[24] Y. V. Bludov, A. Ferreira, N. M. Peres, and M. I. Vasilevskiy, "A primer on surface plasmon-polaritons in graphene," International Journal of Modern Physics B, vol. 27, no. 10, 1341001, 74 pages, 2013.

[25] N. M. R. Peres, Y. V. Bludov, J. E. Santos, A.-P. Jauho, and M. I. Vasilevskiy, "Optical bistability of graphene in the terahertz range," Physical Review B: Condensed Matter and Materials Physics, vol. 90, no. 12, Article ID 125425, 2014.

[26] S. A. Mikhailov and K. Ziegler, "Nonlinear electromagnetic response of graphene: frequency multiplication and the selfconsistent-field effects," Journal of Physics: Condensed Matter, vol. 20, no. 38, p. 384204, 2008.

[27] T. Zhan, X. Shi, Y. Dai, X. Liu, and J. Zi, "Transfer matrix method for optics in graphene layers," Journal of Physics: Condensed Matter, vol. 25, no. 21, Article ID 215301, 2013. 

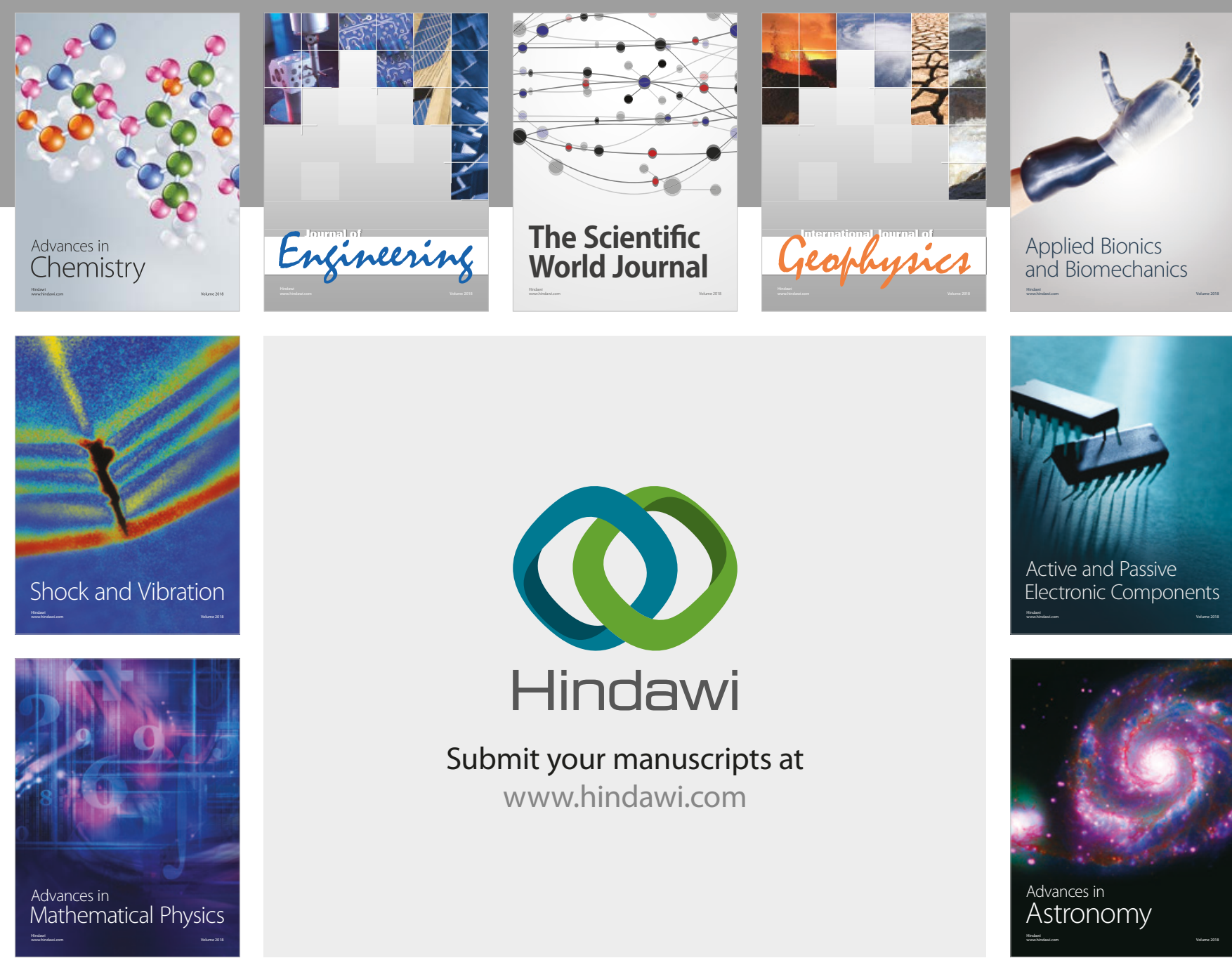

Submit your manuscripts at

www.hindawi.com

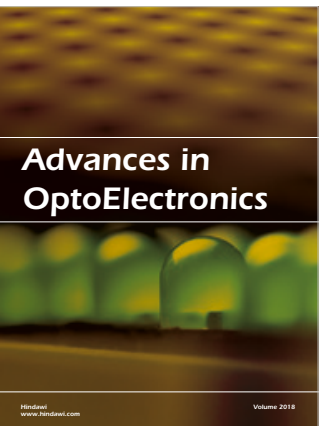

\section{Rotcting Machinery}
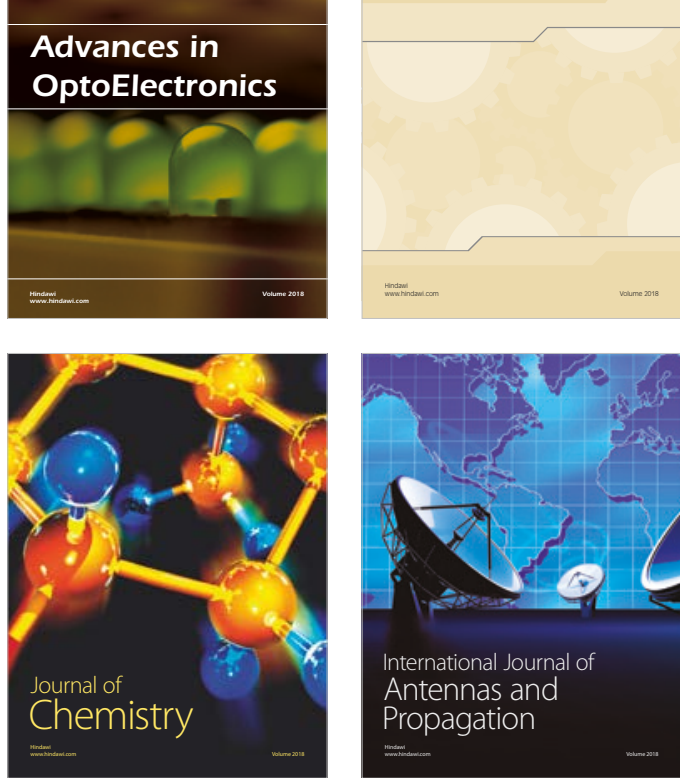

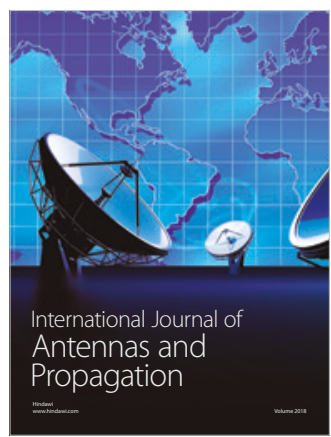

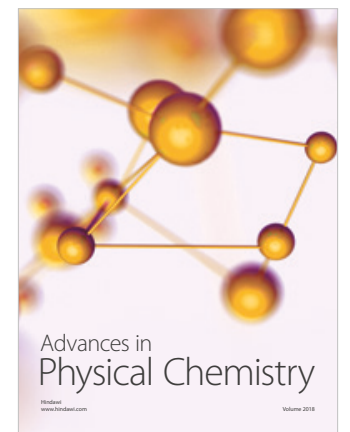

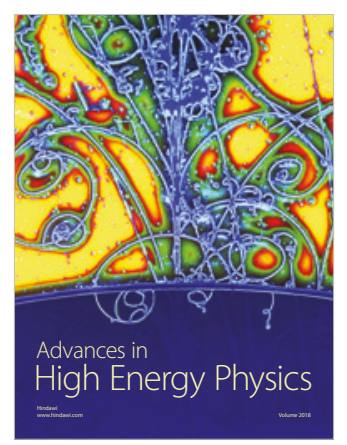

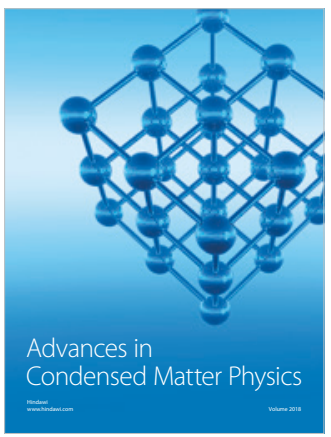

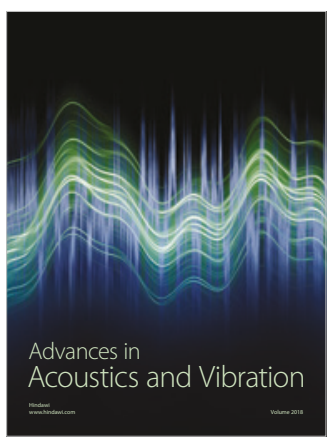

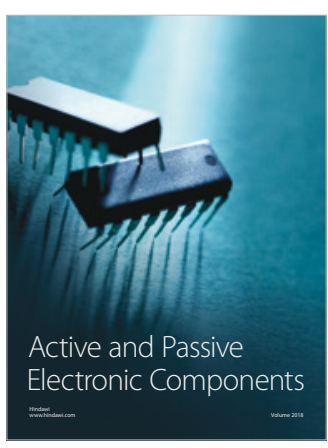
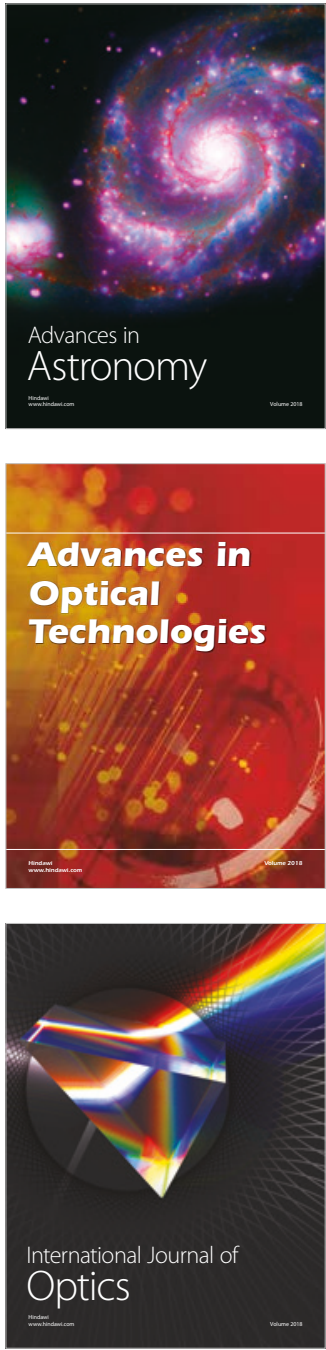